\title{
Analytic Guidance for the First Entry in a Skip Atmospheric Entry
}

\author{
Eduardo García-Llama* \\ GB Tech, Inc., Houston, Texas, 77058, USA
}

\begin{abstract}
This paper presents an analytic method to generate a reference drag trajectory for the first entry portion of a skip atmospheric entry. The drag reference, expressed as a polynomial function of the velocity, will meet the conditions necessary to fit the requirements of the complete entry phase. The generic method proposed to generate the drag reference profile is further simplified by thinking of the drag and the velocity as density and cumulative distribution functions respectively. With this notion it will be shown that the reference drag profile can be obtained by solving a linear algebraic system of equations. The resulting drag profile is flown using the feedback linearization method of differential geometric control as guidance law with the error dynamics of a second order homogeneous equation in the form of a damped oscillator. This approach was first proposed as a revisited version of the Space Shuttle Orbiter entry guidance. However, this paper will show that it can be used to fly the first entry in a skip entry trajectory. In doing so, the gains in the error dynamics will be changed at a certain point along the trajectory to improve the tracking performance.
\end{abstract}

\section{Nomenclature}

\author{
Acronyms \\ $C E V \quad$ Crew Exploration Vehicle \\ EI Entry Interface \\ NSEG Numeric Skip Entry Guidance \\ Symbols \\ (') Time Derivative \\ ()' Derivative With Respect to Velocity \\ $\langle\cdot\rangle \quad$ Mean Value \\ Subscript \\ $i \quad$ Initial \\ $f \quad$ Final (or Exit) \\ $r \quad$ Reference
}

\section{Introduction}

A capsule vehicle such as the one considered in this paper, in the 0.3 to 0.4 lift to drag ratio $(L / D)$ class, returning from the Moon can fly a maximum of about $3700 \mathrm{Km}$. (2000 nmi) downrange using an Apollo like direct entry. In order to fly longer ranges the capsule trajectory must be lofted to decrease aerodynamic forces. Lofting, or Up-Control, implies using the vehicle lift to push it out of the atmosphere and slow the rate at which energy is dissipated. During this high-altitude and low-drag skip phase of the entry, the vehicle can dramatically increase its range capability. The crew flying on such a trajectory will experience a short exo-atmospheric phase before the second entry. ${ }^{1}$

This paper is focused on entries from velocities above circular velocities that require a skip phase to achieve the range necessary to reach the intended destination. Rather than using predictive or optimization

\footnotetext{
${ }^{*}$ Contractor to NASA Johnson Space Center, Aeroscience and Flight Mechanics Division/EG5, 2101 Nasa Parkway, Professional Member.
} 
methods, this paper explores the possibility of generating analytically a reference drag-vs-velocity profile for the first entry in a skip entry trajectory. The fundamental idea of an analytic development of a drag reference profile for entry is an approach already conceived for the Space Shuttle Orbiter guidance logic ${ }^{2}$ and is based on the fact that the range to be flown during entry is a unique function of the drag acceleration maintained throughout the flight. This range is predictable using analytic techniques for simple geometric drag acceleration functions of the relative velocity, provided the local flight path angle is near zero. For low speeds, where the flight path angle is not sufficiently near zero, range predictions can be analytically computed for simple drag acceleration functions if the independent variable is changed from relative velocity to energy with respect to the Earth. Flight throughout the entry corridor can be achieved by linking these geometric functions together in a series. For the Space Shuttle, five basic drag reference segments were selected. Two quadratic segments are used at high speeds, a pseudoequilibrium glide segment and a constant segment in the intermediate speed region and a linear segment, which is a function of energy at low speeds.

In the case of a first entry in a skip entry, the complete drag geometric function for the entire phase can not be in the form of a quadratic polynomial since this would imply equal derivatives of the drag at equal drag levels. We will see later that the derivative of the drag is intimately related to the flight path angle and having, for example, the same drag derivative at entry and at exit would be incompatible with satisfying entry and exit conditions on the flight path angle. In this paper we propose to express the drag reference as a polynomial of the velocity with degree higher than two. The degree will be related to the number of equations needed to generate a reference profile, that is, to meet the conditions necessary to fit the requirements of the complete entry phase. Also, since the portion we are interested in is the first entry in a skip entry, the velocities involved will be hypersonic with small flight path angles. Consequently, there will be no need to change the independent variable to energy.

Meeting the conditions necessary to fit the requirements of the complete entry phase basically means that it is assumed that the preestablished skipout conditions satisfy the range requirement of the whole entry. Although this aspect of the mission planning is not going to be addressed in this paper, the assumption is based on the following rationale: Once the landing site and entry interface are known, if, for instance, the Apollo guidance is used as the guidance logic for the second entry, there is a set of initial conditions required for the initiation of that second entry. Those initial conditions, a triplet formed by the range to go, velocity $(V)$ and flight path angle $(\gamma)$, can be chosen to be in the middle of the performance envelope of the Apollo guidance. As a first approximation, $(V,-\gamma)$ would constitute the skipout conditions in the first entry, and by integrating backwards through the Kepler phase the range that must be covered from entry interface can be determined. The process of finding the target skipout conditions can be further refined. Therefore, for a given entry interface and landing site, it is possible in principle to define a target set for the skipout or exit conditions in terms of the relative velocity, flight path angle and range to be covered from entry interface.

The generic method proposed to obtain the drag reference profile will be further simplified by thinking of the drag as a density function of the velocity or, conversely, by thinking of the velocity as the cumulative distribution function of the drag. With this notion it will be shown that the reference drag profile can be generated by solving a linear algebraic system of equations.

The trajectories generated with this method will be tracked through the implementation of the feedback linearization method of differential geometric control as a guidance law with the error dynamics of a second order homogeneous equation in the form of a damped oscillator. This type of guidance law is detailed in Ref. 3, which demonstrates its commonality with the Shuttle entry guidance. In the case of the Space Shuttle, the drag geometric functions of the segments described above justify that its entry guidance was conceived as a triple integrator since that guarantees perfect tracking of a drag expressed up to a quadratic function of the velocity. In contrast, its revisited version, in Ref. 3, is presented as a double integrator with proportional and derivative feedback terms in its error dynamics. This is so because the main purpose of that work was to show that the approach taken in the original derivation of the Shuttle entry guidance had a lot in common with the, at that time, recently developed, feedback linearization method of differential geometric control; and the difference between the two laws were more clearly identified by neglecting few features in both guidance laws, like the addition of an integral feedback term as the one actually present in the Shuttle entry guidance.

The guidance law, as presented in Ref. 3, in the form of a double integrator, guarantees perfect tracking of a drag function in the form of, at most, a linear function of the velocity or, equivalently, of a drag in a quadratic form with a constant error in steady state. However, in this paper it is going to be shown that using such an entry guidance will allow to track the first entry of a skip entry trajectory with the drag in 
the geometric form of a polynomial of the velocity with degree higher than two.

As we will see later, the generated reference drag profiles, although polynomials of the velocity of degree 5, at low velocities will approximate a linear function of the velocity, which can be tracked with zero steady state error with a double integrator. It remains to be seen the performance of such controller at high velocities, where the drag profile does not necessarily behave as a polynomial of smaller degree. However, although presumably a triple integrator might result in a better performance at such high velocities, we could also suspect that the fast dynamics at high velocities could present almost the same challenges to either double or triple integrator. It will be shown that satisfactory performance is achieved using the double integrator control scheme.

Also, we will see that, in contrast to what is presented in Ref 3 and to improve the tracking performance, in our case the coefficients of the damped oscillator equation will not remain constant throughout the flight. The control gains will be changed depending on the velocity region and the curvature of the drag as a function of the velocity.

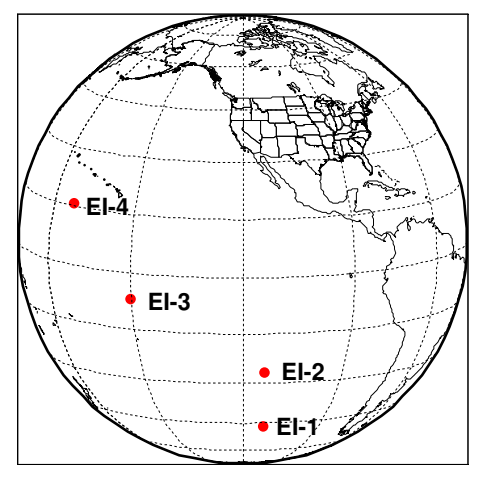

Figure 1. Location of the Test Entry Interfaces With Respect to the U.S.

A number of different approaches to skip entry guidance for the Orion CEV spacecraft have been under evaluation at the Flight Mechanics and Trajectory Design Branch at the NASA's Johnson Space Center. Currently, a guidance using predicted capabilities by a numerical approach that uses multiple trajectory propagations to determine a bank command is providing the most reliable means of meeting the skip entry range requirement. This numerical algorithm, called NSEG, combines features of the original Apollo Guidance algorithm with a numerical scheme for computing a real-time long-range skip trajectory. ${ }^{1}$

Out of a comprehensive set of 60,000 skip entry cases (20 nominal and 59,980 dispersed) that have been simulated for the CEV using NSEG, we are going to use the 20 nominal ones as test cases for this paper. As it was explained before, since the means to obtain the exit conditions from the knowledge of the landing site and entry interface is not going to be addressed in this paper, our initial and final test conditions will be those pertaining to these 20 nominal trajectories. The 20 cases are subdivided in 4 groups. Each group is composed of 5 trajectories that have a common entry interface (figure 1) but different target landing sites in the west continental United States and different $L / D s(0.3,0.33$ and 0.35$)$.

As it is explained in Ref 1 , the flight path angle at entry interface is defined by the skip entry flight corridor that enables satisfying all the mission design considerations, like acceleration magnitudes and durations, service module disposal, heat rate and heat load constraints, landing site precision and safe landing after a failure. Thus, no investigation on the trajectory constraints will be presented in this paper, since we are going to use as initial flight path angles those of the 20 nominal test cases run with NSEG.

\section{Equations of Motion}

The Earth-relative longitudinal translational state of the spacecraft is represented by the variables $R$ (range), $h$ (altitude above the Earth's surface), $V$ (Earth relative velocity) and $\gamma$ (Earth relative flight path angle). The equations of motion in this document use a coordinate system with one axis oriented along the Earth-relative velocity vector, one axis perpendicular to the plane formed by the position and Earth-relative velocity vectors, and a third axis completing the right-hand coordinate system. The equations of motion are 
as follows

$$
\begin{gathered}
\dot{R}=V \cos \gamma \\
\dot{h}=V \sin \gamma \\
\dot{V}=-D-g \sin \gamma \\
\dot{\gamma}=\frac{1}{V}\left[L \cos \phi+\left(\frac{V^{2}}{r_{e}+h}-g\right) \cos \gamma\right]
\end{gathered}
$$

where $r_{e}$ is the mean Earth radius and $g$ is the gravity acceleration. These equations of motion neglect the Coriolis and centripetal accelerations due to Earth's rotation because these accelerations are small compared to the aerodynamic acceleration.

The specific drag and lift are given by

$$
\begin{aligned}
& D=\rho V^{2}\left(\frac{S_{r} C_{D}}{2 m}\right) \\
& L=\rho V^{2}\left(\frac{S_{r} C_{L}}{2 m}\right)
\end{aligned}
$$

where $S_{r}$ and $m$ are the reference surface and mass of the vehicle respectively. The drag and lift aerodynamic coefficients $\left(C_{D}\right.$ and $\left.C_{L}\right)$ are assumed constant since only hypersonic velocities are going to be involved in the phase of interest.

An exponential atmospheric density model with constant atmospheric density at base altitude is assumed for this study

$$
\rho=\rho_{0} e^{-\frac{h}{h_{s}}}
$$

where $h_{s}$ is the atmospheric density scale height.

The translational state is controlled by adjusting the vertical lift-to-drag ratio $(L / D) \cos \phi$. Equivalently, the bank angle $\phi$ is taken to be the control in the following analysis.

\section{Generation of the Reference Drag Trajectory}

The phase under consideration is the first entry in a skip atmospheric entry. The origin for this phase is an initial velocity and flight path angle, and its destination is a set of specific exit conditions in terms of velocity and flight path angle at a desired range.

The idea behind this method is, first, to assume a reference drag trajectory as a polynomial expression of the velocity

$$
D(V)=\sum_{n=0}^{m} a_{n} V^{n}
$$

and, second, to resolve the $m+1$ equations required to determine the coefficients $a_{n}$.

Five equations can be considered as basic to generate a drag reference profile: four equations establishing the initial and final (or exit) conditions on velocity and flight path angle, and a fifth equation establishing the condition on the range flown.

The four equations relating the initial and final conditions are obtained as follows: Two equations relate the initial and final velocities to the initial and final drags

$$
D_{i, f} \equiv D\left(V_{i, f}\right)=\sum_{n=0}^{m} a_{n} V_{i, f}^{n}
$$


In order to find the relation between the drag and the flight path angle we need to make use of the equation for the atmospheric density. Differentiating Eq. (7) with respect to time the following equation is obtained

$$
\frac{\dot{\rho}}{\rho}=-\frac{\dot{h}}{h_{s}}
$$

On the other hand, by differentiating Eq. (5) with respect to time and dividing by $D$ results

$$
\frac{\dot{D}}{D}=\frac{\dot{\rho}}{\rho}+\frac{2 \dot{V}}{V}
$$

Now, combining Eqs. (10 and 11) results

$$
\frac{\dot{D}}{D}+\frac{2 D}{V}=-\frac{\dot{h}}{h_{s}}
$$

where it has been assumed that the drag coefficient, $C_{D}$, does not change with time. Using the equations of motion, the relation between the altitude rate and the flight path angle is known, thus, we need to know how the time derivative of the drag is related to the expression of the drag as a function of the velocity

$$
\frac{\dot{D}}{D}=\frac{1}{D} \frac{d D}{d t}=\frac{1}{D} \frac{d D}{d V} \frac{d V}{d t}=-\frac{d D}{d V}
$$

where small flight path angles have been assumed since the flight path angle during a typical first entry in a skip entry is sufficiently small as to approximate the $\sin \gamma$ to 0 , thus $\dot{V} \approx-D$. Substituting Eq. (13) in Eq. (12) results

$$
-\frac{d D}{d V}+\frac{2 D}{V}=-\frac{V \sin \gamma}{h_{s}}
$$

Hence, the other two equations relating the initial and final velocities and flight path angles to the initial and final drags are

$$
\left.D_{i, f}^{\prime} \equiv \frac{d D}{d V}\right|_{i, f}=\sum_{n=1}^{m} n a_{n} V_{i, f}^{n-1}=\frac{V_{i, f} \sin \gamma_{i, f}}{h_{s}}+\frac{2 D_{i, f}}{V_{i, f}}
$$

A specific range $R$ must be covered between the initial and final conditions. For small flight path angles combining Eqs. (1 and 3), separating variables and integrating yields

$$
R=-\int_{V_{i}}^{V_{f}} \frac{V}{D(V)} d V
$$

Having the drag expressed as a polynomial of the velocity implies that Eq. (16) can be solved analytically, and therefore the last of the five basic equations, the range equation, will be given by

$$
R=\sum_{j=1}^{m} \frac{r_{j}}{\sum_{n=1}^{m} n a_{n} r_{j}^{n-1}} \log \left(\frac{V_{i}-r_{j}}{V_{f}-r_{j}}\right)
$$

where $r_{j}$ are the roots of $D(V)$.

However, the equation for range can be simplified by transforming it into an equation in terms of time. If a mean drag is associated to the phase under consideration, integrating Eq. (16) results

$$
\langle D\rangle=\left(V_{i}^{2}-V_{f}^{2}\right) / 2 R
$$

Also, from the mean of the drag, the time duration of this phase can be found

$$
\Delta T=-\Delta V /\langle D\rangle
$$

Now that $\Delta T$ is known, since $\dot{V} \approx-D$, the range equation in terms of time can be calculated 


$$
\Delta T=-\int_{V_{i}}^{V_{f}} \frac{1}{D(V)} d V=\sum_{j=1}^{m} \frac{1}{\sum_{n=1}^{m} n a_{n} r_{j}^{n-1}} \log \left(\frac{V_{i}-r_{j}}{V_{f}-r_{j}}\right)
$$

From a computational stand point, Eq. (20) is better suited than Eq. (17) due to the absence of the terms $r_{j}$ in the numerator.

In summary, by solving the system composed of Eqs. 9, 15 and 20, a drag reference profile expressed as a degree $4(m=4)$ polynomial of the velocity can be generated. Appendix A provides more detail on how to obtain the coefficients of the drag polynomial when one of the equations is in the form of Eq. (20).

\section{A. Alternate Method for Range Equation}

The equation on range, Eq. (20), implies that numerical methods need to be used to find at least one of the coefficients in the drag polynomial (see Appendix A). It would be highly desirable to find a relation such that the set of equations to obtain the coefficients of the drag polynomial could be solved as a linear algebraic system. This section presents a method to generate a substitute to Eq. (20) such that the system containing the five basic equations becomes a linear algebraic system.

One way to achieve the remaining algebraic equation is by relating the range to the integral of the drag along the velocity

$$
\int_{V_{f}}^{V_{i}} D(V) d V=\sum_{n=1}^{m} \frac{a_{n}}{n}\left(V_{f}^{n}-V_{i}^{n}\right)
$$

The problem is that, in principle, we only know how to relate range and drag through Eq. (16). The following steps are proposed to achieve the desired algebraic relation. We know that

$$
d V=-D d t
$$

Multiplying Eq. (22) by the drag and integrating results

$$
\int_{V_{i}}^{V_{f}} D d V=-\int_{t_{i}}^{t_{f}} D^{2} d t
$$

Expressing the integral in the second member of Eq. (23) in terms of means and increments yields

$$
\int_{V_{i}}^{V_{f}} D d V=-\left\langle D^{2}\right\rangle \Delta T
$$

The mean of the drag and in turn the time duration can be calculated from Eqs. (18 and 19). However, the mean of the square of the drag must be calculated in order to find the integral of interest. The next method is proposed to find $\left\langle D^{2}\right\rangle$.

The relation between velocity, drag and time in Eq. (22) may allow us to think of the velocity as the cumulative distribution function related to the drag, which we can think of as a density function. Note that a density function $f$ is defined in terms of its distribution function $F$ as ${ }^{4}$

$$
f(x)=d F(x) / d x
$$

Therefore, the relation between velocity and drag could be understood as the relation between a cumulative distribution function and its density function that have been specifically scaled and initialized. For instance, if it was not for the negative sign, the velocity cumulative distribution of the drag density would be very similar to the Maxwell distribution and density functions. Figure 2 shows a comparison of actual data from the simulation of the first entry portion of a skip entry flight to that generated by a Maxwell density and distribution functions that have been properly scaled and initialized for comparison purposes.

Let us assume that the drag in terms of time can be considered as a density function. In that case, a function expressing the variance, $\sigma^{2}$, in terms of $\langle D\rangle^{2}$ could exist, as it is the case, for example, in the

Maxwell distribution, where $\sigma^{2} \propto \mu^{2}$ with $\mu$ being the mean value. ${ }^{4}$ If such relation exists, then $\left\langle D^{2}\right\rangle$ could be easily found since

$$
\left\langle D^{2}\right\rangle=\sigma^{2}+\langle D\rangle^{2}
$$




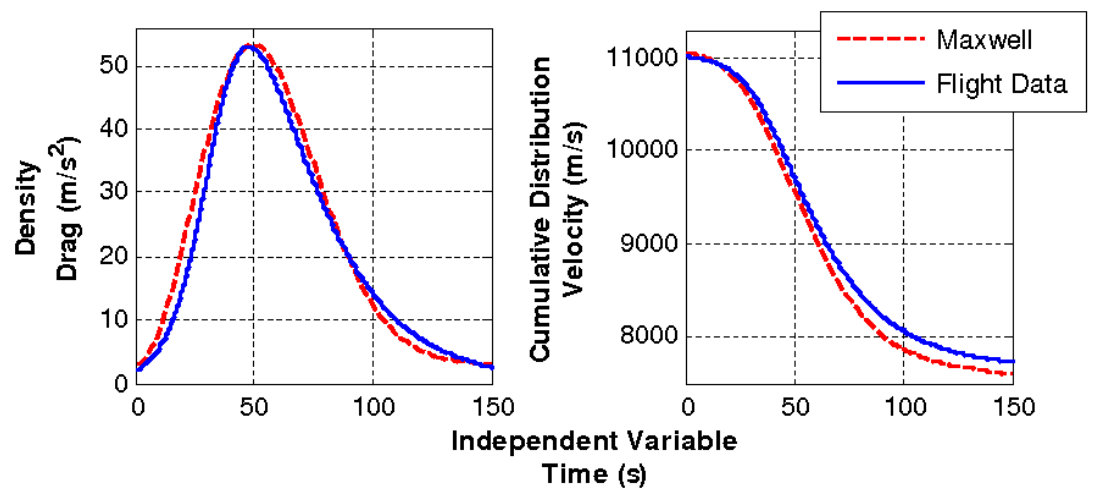

Figure 2. Example of Drag and Velocity Signals During the First Entry in a Skip Entry Compared to Maxwell Density and Distribution Functions.

Ultimately, the relation between $\left\langle D^{2}\right\rangle$ and $\langle D\rangle^{2}$ could be as simple as a linear function. From our 20 test cases, referred to in the introduction, we may try to find empirically the relationship between $\left\langle D^{2}\right\rangle$ and $\langle D\rangle^{2}$, and check if it follows a specific relation. Figure (3) presents that relation. A data correlation coefficient of 0.998 for the 20 nominal cases shows that $\left\langle D^{2}\right\rangle$ and $\langle D\rangle^{2}$ are, in fact, highly correlated (this analysis was also carried out for the 59,980 dispersed cases. For the dispersed cases, the correlation coefficient was found to be 0.985$)$.

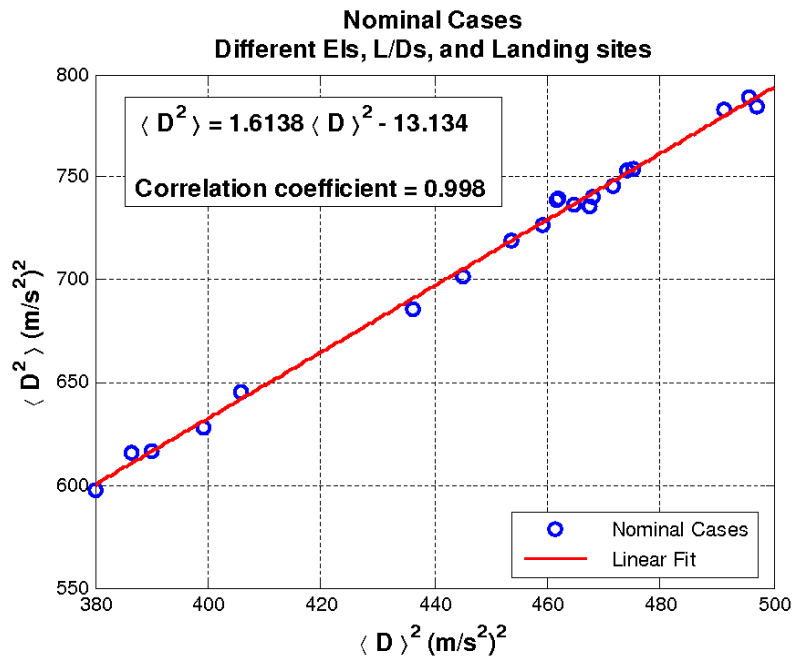

Figure 3. Relation Between $\left\langle D^{2}\right\rangle$ and $\langle D\rangle^{2}$ in the Phase of Interest for 20 Nominal Skip Entries Flown Using NSEG.

From figure (3) we deduce that

$$
\left\langle D^{2}\right\rangle=k_{d 1}\langle D\rangle^{2}+k_{d 2}
$$

and the range equation, Eq. (20), can now be substituted by

$$
\sum_{n=1}^{m} \frac{a_{n}}{n}\left(V_{f}^{n}-V_{i}^{n}\right)=\left(k_{d 1}\langle D\rangle^{2}+k_{d 2}\right) \Delta T
$$

where the range is indirectly accounted through the terms $\langle D\rangle$ and $\Delta T$.

\section{B. Constraint Equations}

The five basic equations with which a drag reference profile can be generated have been derived. If only these five equations were considered then our drag polynomial should have degree $4(m=4)$ to have five 
unknown coefficients $\left(a_{0} \ldots a_{4}\right)$. Should we be interested in adding constraint equations to the drag profile, the degree of the drag polynomial should increase accordingly.

In this section, one additional constraint equation is going to be generated. From the equations derived so far, the drag reference trajectory may turn out to have a maximum drag acceleration at an arbitrary velocity. We are interested in generating drag reference trajectories whose shape is more in line with realistic shapes flown in the thousands of skip entry cases already simulated using NSEG. From all those cases, a quite simple trend can be found by inspection of the drag profiles: the velocity at which the maximum drag occurs has a high correlation with the product of the initial and final velocities (figure 4). The correlation factor found is 0.991 for our 20 nominal test cases (this analysis was also carried out for the 59,980 dispersed cases. For the dispersed cases, the correlation coefficient was found to be 0.851).

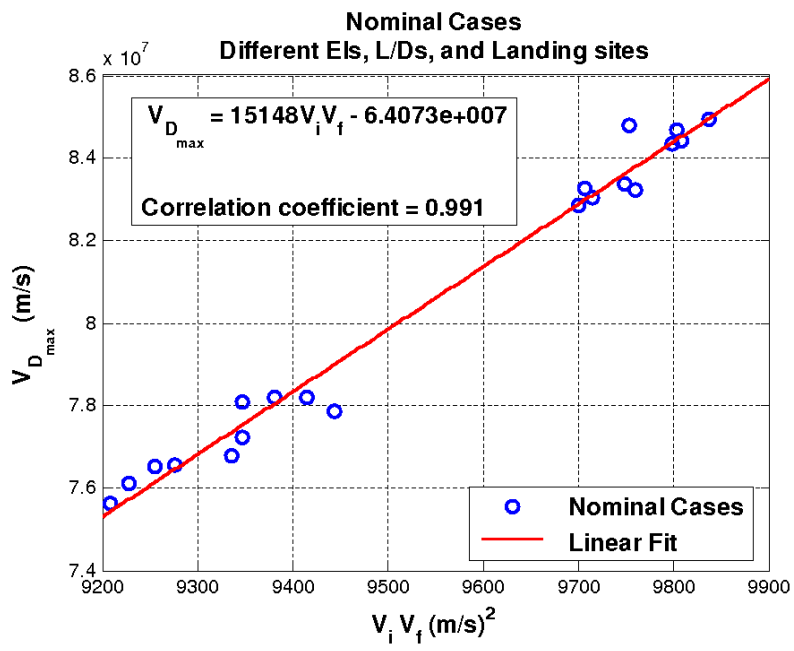

Figure 4. Relation Between the Velocity at Maximum Drag and the Product of the Initial and Final Velocities in the Phase of Interest for 20 Nominal Skip Entries Flown Using NSEG.

Therefore, our constraint equation will be given by

$$
D_{D_{\max }}^{\prime}=\sum_{n=1}^{m} n a_{n} V_{D_{\max }}^{n-1}=0
$$

where the velocity at maximum drag, $V_{D_{\max }}$, can be expressed as

$$
V_{D_{\max }}=k_{v 1} V_{i} V_{f}+k_{v 2}
$$

\section{Summary and Results}

From the results obtained in the previous subsections, the algebraic system of 5 or 6 linear equations (depending on whether the drag is expressed as a 4 degree or 5 degree polynomial respectively) from where the reference drag trajectories can be obtained is

$$
\left(\begin{array}{lllllll}
1 & V_{i} & V_{i}^{2} & V_{i}^{3} & V_{i}^{4} & V_{i}^{5} \\
1 & V_{f} & V_{f}^{2} & V_{f}^{3} & V_{f}^{4} & V_{f}^{5} \\
0 & 1 & 2 V_{i} & 3 V_{i}^{2} & 4 V_{i}^{3} & 5 V_{i}^{4} \\
0 & 1 & 2 V_{f} & 3 V_{f}^{2} & 4 V_{f}^{3} & 5 V_{f}^{4} \\
V_{f}-V_{i} & \frac{V_{f}^{2}-V_{i}^{2}}{2} & \frac{V_{f}^{3}-V_{i}^{3}}{3} & \frac{V_{f}^{4}-V_{i}^{4}}{4} & \frac{V_{f}^{5}-V_{i}^{5}}{5} & \frac{V_{f}^{6}-V_{i}^{6}}{6} \\
--- & --- & --- & --- & --- & --- \\
0 & 1 & 2 V_{D_{\max }} & 3 V_{D_{\max }}^{2} & 4 V_{D_{\max }}^{3} & 5 V_{D_{\max }}^{4}
\end{array}\right)\left(\begin{array}{c}
a_{0} \\
a_{1} \\
a_{2} \\
a_{3} \\
a_{4} \\
-- \\
a_{5}
\end{array}\right)=\left(\begin{array}{c}
D_{i} \\
D_{f} \\
\frac{V_{i} \sin \gamma_{i}}{h_{s}}+\frac{2 D_{i}}{V_{i}} \\
\frac{V_{f} \sin \gamma_{f}}{h_{s}}+\frac{2 D_{f}}{V_{f}} \\
c_{1} c_{2}\left\langle D^{2}\right\rangle \Delta T \\
------ \\
0
\end{array}\right)
$$

In the second member of Eq. (31), $c_{1}$ is an empirically derived constant that accounts for the error resulting from using the $\langle D\rangle$ calculated with Eq. (18) instead of the actual $\langle D\rangle$ that was being used to 
generate $k_{d 1}$ and $k_{d 2}$ in Eq. (27). From the results shown in figure 5, we can deduce that $c_{1}=1.043$. This is in itself an interesting result. It shows a linear dependence between the actual $\langle D\rangle$ and the $\langle D\rangle$ calculated with Eq. (18).

The constant $c_{2}$ (also in the second member of Eq. (31)) is a correction, or calibration, factor empirically derived to minimize the mean of the absolute value of the range errors found in the set of 20 reference trajectories generated using Eq. (31) when the obtained ranges are compared to the actual ones of the considered 20 nominal trajectories simulated using NSEG $\left(c_{2}=1.056\right)$.

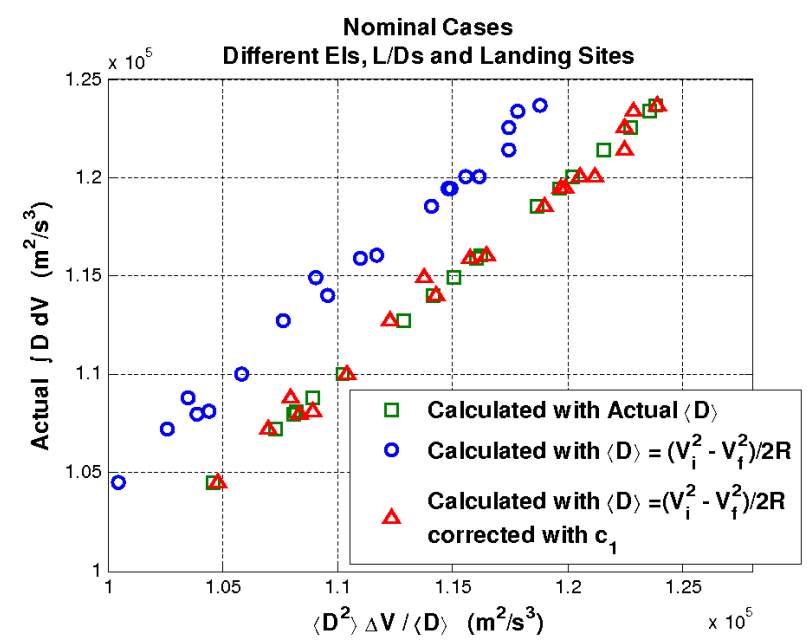

Figure 5. Comparison of the Results Obtained for the Integral of the Drag Along the Velocity When the Actual $\langle D\rangle$, the Calculated $\langle D\rangle$ and the Empirically Corrected Formula are Used.

Figure 6 shows the degree 5 drag reference trajectories generated using Eq. (31) when the range and initial and final conditions of the 20 nominal test cases considered in this document are used. With generality, a skip entry is defined whenever the Up-Control acceleration drops below $0.2 g$ during the Up-Control phase of the flight, therefore, the final drag was chosen to be $0.2 g$. For simplicity, in the generation of the trajectories shown in figure (6), the initial drag was set equal to the final drag $\left(D_{i}=D_{f}=0.2 g\right)$.

The range error subplot in figure 6 shows that this method provides for range errors smaller than $1 \%$ in magnitude, with an average value of $0.47 \%$.
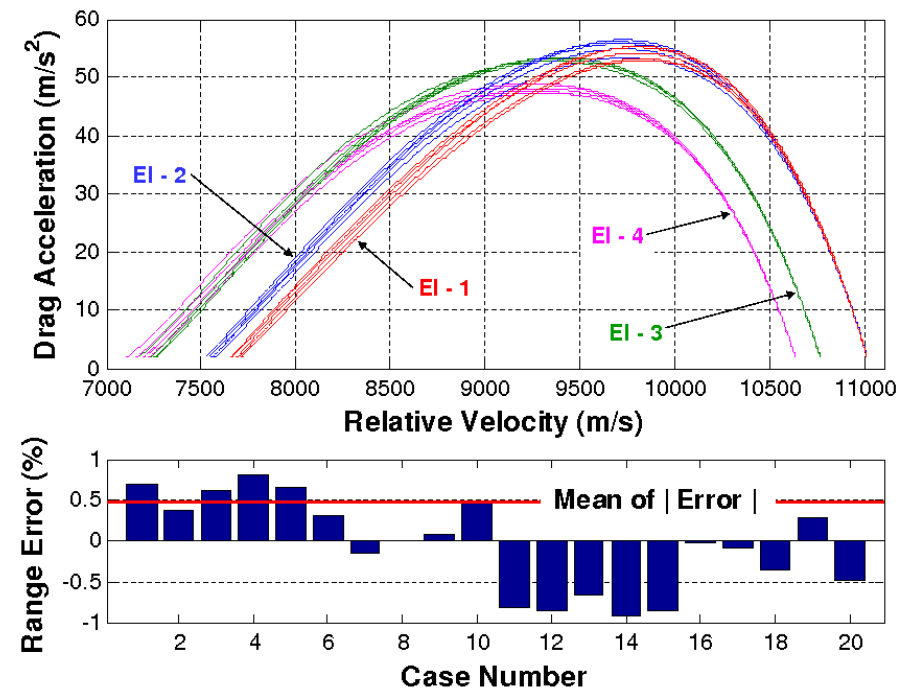

Figure 6. Drag Reference Trajectories Generated Using Eq. (31) and Resulting Range Errors with Respect to the Actual Ranges of the 20 Nominal Skip Entry Cases Flown Using NSEG. 


\section{Feasible Trajectory Generation}

Given a reference trajectory $D(V)$, is it possible to know, a priori, that it is feasible? That is, can we guarantee the existence of a bank control command such that $D(V)$ can be tracked? The approach to derive the reference bank control consists of time differentiating the drag along the trajectory (i.e., taking the Lie derivative of the drag) until the first appearance of the control. This has been done in references 2 and 3 , and the reference bank angle can be expressed as

$$
\phi=\arccos \left\{\frac{\ddot{D}-\dot{D}\left(\frac{\dot{D}}{D}-\frac{3 D}{V}\right)+\frac{4 D^{3}}{V^{2}}+\frac{D}{\left\langle h_{s}\right\rangle}\left(\frac{V^{2}}{r_{e}+\langle h\rangle}-\langle g\rangle\right)}{-\frac{D^{2}}{(L / D)\left\langle h_{s}\right\rangle}}\right\}
$$

where the mean values of $g, h_{s}$ and $h$ have been used.

Equation (13) shows the time derivative of the drag in terms of the drag and the derivative of the drag with respect to the velocity. In order to find the second time derivative of the drag, to be substituted in Eq. (32), we need to differentiate Eq (13) a second time. This operation results in the following expression for $\ddot{D}$

$$
\ddot{D}=D\left(\frac{d D}{d V}\right)^{2}+D^{2} \frac{d^{2} D}{d V^{2}}
$$

The resulting bank angle profiles, corresponding to the drag reference trajectories depicted in figure (6), are shown in figure (7) grouped by entry interface. A saturation can be appreciated at the beginning of the trajectory for a small velocity interval, when the drag is still fairly low. The following section will show how this feature is dealt with when the guidance law is implemented. Besides this undesired feature, the bank profiles remain completely feasible.

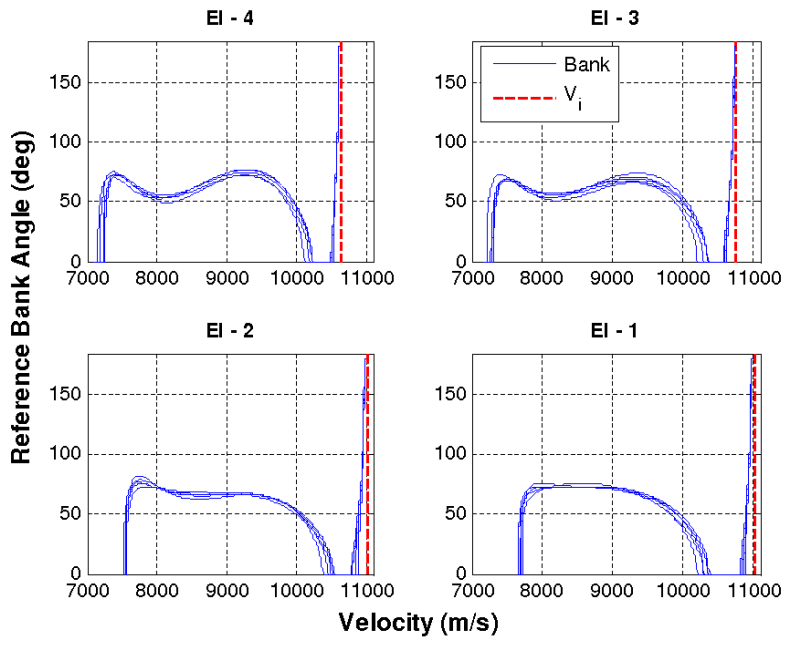

Figure 7. Reference Bank Angle Profiles Associated to the Drag Reference Trajectories Generated Using Eq. (31).

\section{Reference Trajectory Tracking}

Once the reference drag vs velocity trajectory is chosen, the bank-angle control law must be developed for tracking the reference trajectory. The tracking control law we are going to use to fly the drag profiles shown in figure (6) is the guidance law for the Space Shuttle Orbiter revisited using nonlinear geometric methods, with the error dynamics of a second order homogeneous equation in the form of a damped oscillator. This work is detailed in reference 3 and, therefore, only a summary of its theoretical development and background is going to be presented here.

The approach to deriving the bank-angle control law begins with time differentiating the drag along a trajectory (i.e., taking the Lie derivative of the drag) until the first appearance of the control. The first derivative is obtained by differentiating Eq. (5) 


$$
\dot{D}=-\frac{D V}{\left\langle h_{s}\right\rangle} \sin \gamma-\frac{2 D^{2}}{V}
$$

and the second derivative is given by

$$
\ddot{D}=a(V, D, \dot{D})+b(V, D, \dot{D}) u
$$

where

$$
\begin{aligned}
& a=\dot{D}\left(\frac{\dot{D}}{D}-\frac{3 D}{V}\right)-\frac{4 D^{3}}{V^{2}}+\left(\langle g\rangle-\frac{V^{2}}{r_{e}+\langle h\rangle}\right) \frac{D}{\left\langle h_{s}\right\rangle} \\
& b=-D^{2} /\left\langle h_{s}\right\rangle \\
& u=(L / D) \cos \phi
\end{aligned}
$$

Equations (34) and (35) have been derived with the approximations $D+g \sin \gamma \approx D$ and $\cos \gamma \approx 1$ and with the mean values of $g, h$ and $h_{s}$.

It is necessary that the transient response is sufficiently fast. It is the reference drag vs velocity trajectory that leads to the desired range at the specified velocity; if the control law does not cause quick enough recovery from perturbations off the reference trajectory, the desired final condition will not be achieved.

Let

$$
\nu=a(V, D, \dot{D})+b(V, D, \dot{D}) u(V, D, \dot{D})
$$

and consider $u$ to be a function of the arguments shown. Defining $\Delta a=a-a_{r}, \Delta b=b-b_{r}$, and $\Delta u=u-u_{r}$, where $a_{r}=a\left(V, D_{r}, \dot{D}_{r}\right)$ and similarly for $b_{r}$ and $u_{r}$, Eq. (37) is rewritten as

$$
\nu=\left(a_{r}+b_{r} u_{r}\right)+\left(\Delta a+\Delta b u_{r}+b \Delta u\right)=\nu_{r}+\Delta \nu
$$

where $\nu_{r}$ and $\Delta \nu$ correspond to the first and second expressions in parentheses, respectively, and $\Delta \nu\left(V, D_{r}, \dot{D}_{r}\right)$ $=0$. Equation (35) becomes

$$
\ddot{D}=\nu_{r}+\Delta \nu
$$

Choosing

$$
\nu_{r}=\ddot{D}_{r}
$$

and defining $\Delta D=D-D_{r}$, the dynamics for the tracking error are given by

$$
\Delta \ddot{D}=\Delta \nu
$$

The feedforward control

$$
u_{r}=\left(1 / b_{r}\right)\left(-a_{r}+\ddot{D}_{r}\right)
$$

obtained by solving Eq. (40) for $u_{r}$ using the relation in Eq. (38) between $\nu_{r}$ and $u_{r}$ thus produces perfect tracking beyond any value of $V$ for which $\Delta D$ and $\Delta \dot{D}$ are both zero. However, if only the feedforward control is used, the resulting time-varying error dynamics are

$$
\Delta \ddot{D}=\Delta a+\Delta b u_{r}
$$

which may not be acceptable. The simplest acceptable error dynamics are of the linear, time-invariant form

$$
\Delta \ddot{D}+2 \zeta \omega \Delta \dot{D}+\omega^{2} \Delta D=0
$$

with positive natural frequency $\omega$ and positive damping ratio $\zeta$, since the error dynamics are then asymptotically (or, more precisely, exponentially) stable with respect to the origin.

In the formalism of the feedback linearization method a nonlinear state transformation and a statedependent control transformation to linearize the input-output dynamics have been used. The state variables $(h, \gamma, V)$ were transformed to $(D, \dot{D}, V)$ and the control was transformed according to Eq. (37). Using the notation $\left(z_{1}, z_{2}, z_{3}\right)=(D, \dot{D}, V)$, the transformed state equations are 


$$
\begin{gathered}
\dot{z}_{1}=z_{2} \\
\dot{z}_{2}=\nu \\
\dot{z}_{3}=-z_{1}+\langle g\rangle\left\langle h_{s}\right\rangle\left(z_{2} z_{3}+2 z_{1}^{2}\right) / z_{1} z_{3}^{2} \\
y=z_{1}
\end{gathered}
$$

The dynamic system between input $\nu$ and output $y$ is linear, time invariant, and in double-integrator form.

Note that by specifying

$$
\nu=a+b u=\Delta \ddot{D}+2 \zeta \omega \Delta \dot{D}+\omega^{2} \Delta D
$$

the desired error dynamics given in Eq. (44) are achieved exactly. The required actual control, obtained by solving Eq. (46) for $u$, is

$$
u=\frac{1}{b_{r}}\left(-a_{r}+\ddot{D}_{r}-\omega^{2} \Delta D-2 \zeta \omega \Delta \dot{D}\right)
$$

Therefore, the commanded bank angle will be given by

$$
\phi_{c}=\arccos \left\{\frac{1}{(L / D) b_{r}}\left(-a_{r}+\ddot{D}_{r}-\omega^{2} \Delta D-2 \zeta \omega \Delta \dot{D}\right)\right\}
$$

\section{A. Test Results}

The performance of the drag tracking control is evaluated on the 20 nominal test cases. At this stage, the same set of control gains will be used in all the simulations regardless of the initial and final conditions. During the controlled simulation, the drag profile is not updated or modified to reduce the range error. Therefore, the target miss accumulated at guidance termination will be a measure of the control performance.

In all simulations, the control starts operating once the initial drag acceleration is higher than $1 \mathrm{~g}$. Prior to that point, the commanded bank angle is constant and equal to $80 \mathrm{deg}$ to avoid the saturation observed in the reference bank angle at the initiation of the entry (figure 7).

Also, the bank angle rate of change and acceleration were limited to $\pm 15 \mathrm{deg} / \mathrm{s}$ and $\pm 6 \mathrm{deg} / \mathrm{s}^{2}$ respectively.

Each controlled simulation is divided in two phases, determined by the velocity and the curvature of the drag as a function of the velocity. Figure 6 shows that the drag becomes almost linear at low velocities. It was determined that changing the set of control gains when the curvature was smaller than a certain threshold resulted in an improvement of the tracking performance. The curvature is defined in Eq. (49), and the curvature threshold was chosen to be -0.05

$$
\kappa=\frac{d^{2} D / d V^{2}}{\left(1+(d D / d V)^{2}\right)^{3 / 2}}
$$

The values of the control gains in phase 1 (high velocities) are $\zeta=0.4$ and $\omega=3 /(80 \cdot \zeta)=0.0938$. In phase 2 (low velocities and $-0.05<\kappa<0$ ) the control gains are $\zeta=0.68$ and $\omega=3 /(25 \cdot \zeta)=0.1765$. Note that for the calculation of $\omega$, the $5 \%$ criteria has been used (settling time equal to 3 times the time constant of the system). Figure 8 shows, for each of the test cases, the absolute range and final flight path angle percentile errors with respect to the values in the 20 nominal cases flown using NSEG.

Figure 8 depicts similar error values for the cases sharing the same entry interface. This can lead us to think that specific sets of control gains can be selected for each group with the same entry interface to improve the tracking performance. Furthermore, the latter could imply that an optimal relation between control gains and the set of initial and final conditions can indeed be found. However, the study of this aspect is not within the scope of this paper and is not going to be addressed here.

Figures 9 and 10 show the results of four controlled trajectories, one from each entry interface. Figure 11 shows the drag error signals for the same set of 4 trajectories. 

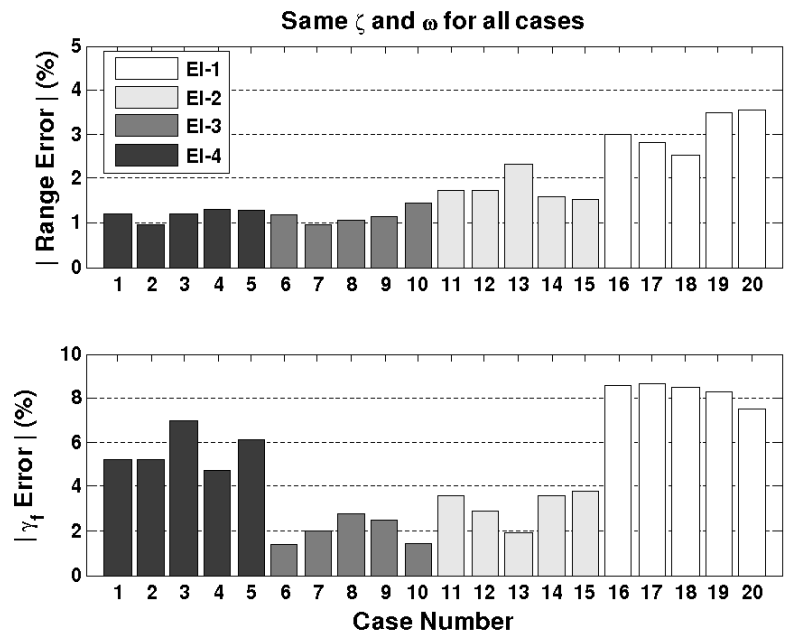

Figure 8. Absolute Range and Final Flight Path Angle Errors of the Controlled Trajectories with Respect to the Values of the 20 Nominal Skip Entry Cases Flown Using NSEG.

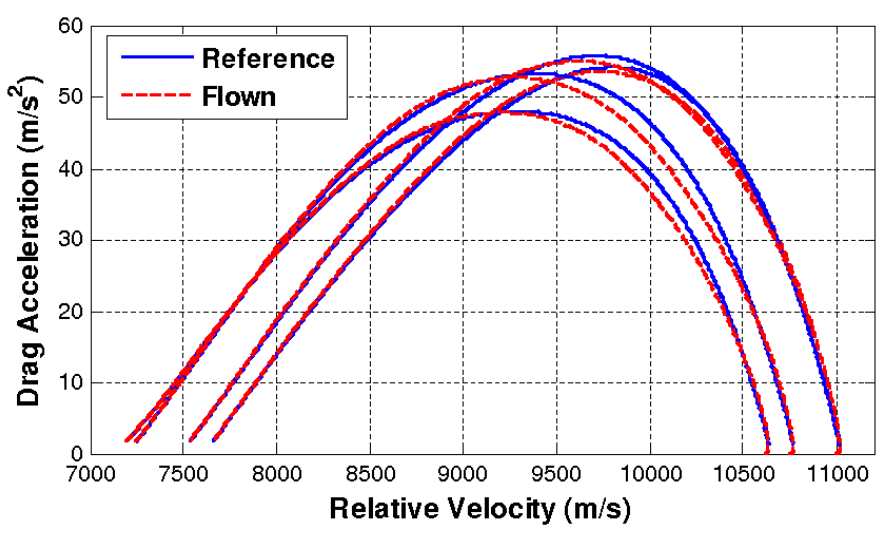

Figure 9. Examples of Drag vs Velocity Trajectories of 4 Controlled Trajectories, One from Each Entry Interface.

\section{Lateral Logic}

In this section the tracking performance when a lateral logic is introduced is evaluated. The initial sign of the bank angle is chosen as to reduce the heading miss with respect to the landing site. A lift vector reversal will be made when a specific crossrange deadband is exceeded. In this study we are using an Apollo-like guidance deadband. ${ }^{5}$ In a first approximation, this deadband is proportional to the capsule's lateral ranging capability at the current velocity and is given by the following relation

$$
Y=\frac{L / D}{24}\left(\frac{V}{V_{\text {sat }}}\right)^{2}
$$

where $V_{\text {sat }}$ is the satellite velocity.

Figure 12 shows the results when the lateral logic is included in the guidance logic. The same set of gains when no lateral logic was included are used now for all the cases as well.

In figure 12, cases 1 to 3 did not require any bank reversal to comply with the deadband as established in Eq. (50). Cases 12, 13 and 16 called for a bank reversal when the velocity was very close to the exit velocity, which allows for very precarious physical capability to satisfy the exit requirements. These last three cases require a more sophisticated lateral logic to prevent such situation. Although bank reversals could have been forced at some point along the trajectory with the purpose of evaluating the lateral logic, it was deemed that the obtained results, as shown in figure 12, suffice to convey the level of tracking performance. In general, 


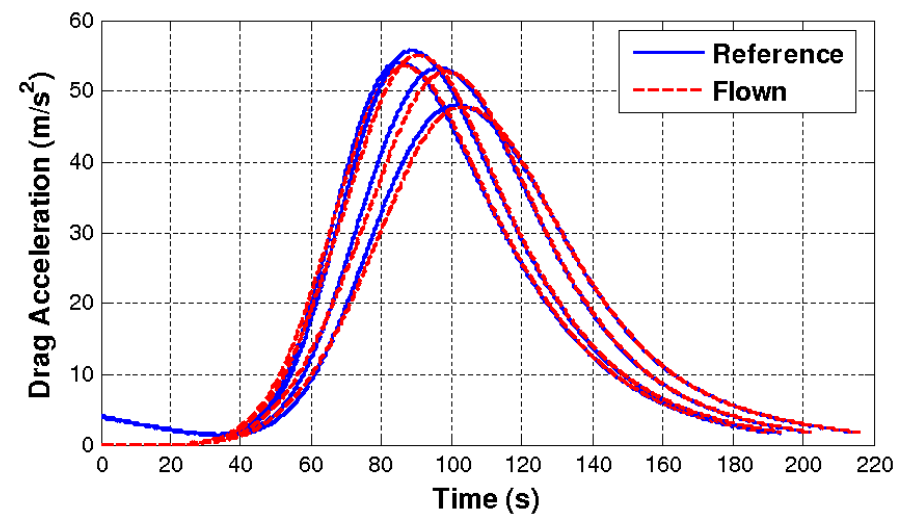

Figure 10. Examples of Drag vs Time Trajectories of 4 Controlled Trajectories, One from Each Entry Interface.

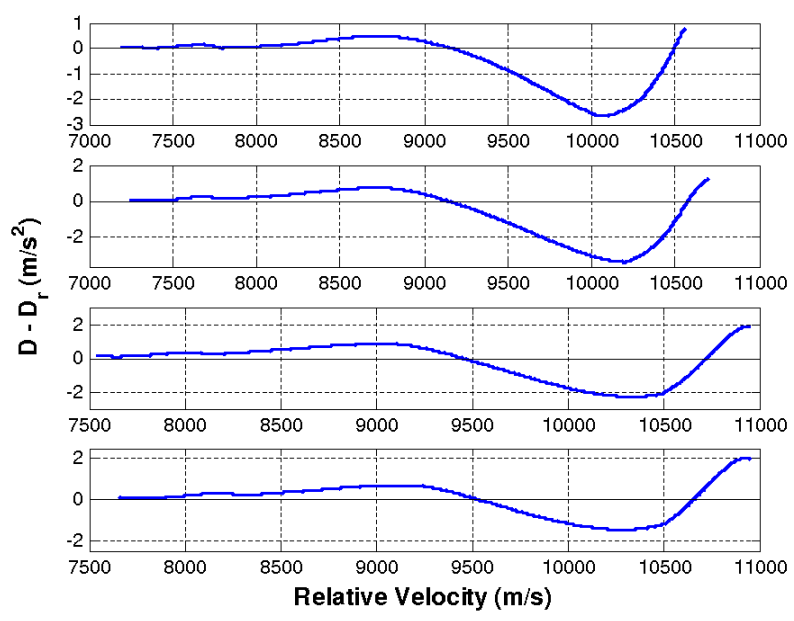

Figure 11. Examples of Drag Acceleration Error Signals of 4 Controlled Trajectories, One from Each Entry Interface.

the results of figure 12 show comparable results to the ones obtained when the lateral logic was not included (figure 8).

\section{Conclusion}

This paper shows that a feasible reference drag trajectory for the first entry portion of a skip atmospheric entry can be generated as a polynomial expression of the velocity. The coefficients of that polynomial are found through the resolution of a system composed of $m+1$ equations, where $m$ is the degree of the drag polynomial. It has been shown that a minimum of five equations (implying a drag polynomial of degree 4) are required to establish the range and the initial and final conditions on velocity and flight path angle. From this foundation, it has been shown that constraints on the trajectory can be imposed through the addition of equations in the system, which must be accompanied by the increase in the degree of the drag polynomial.

In order to simplify the resolution of the system of equations, the notion of the drag being considered as a density function of the velocity, with the velocity as a cumulative distribution function of the drag, has been introduced. Through this notion, together with the introduction of empirically derived constants, it has been shown that the system of equations required to generate the drag profile can be successfully reduced to a system of linear algebraic equations.

The resulting drag profiles have been flown using the feedback linearization method of differential geometric control as a guidance law with the error dynamics of a second order homogeneous equation in the form of a damped oscillator. Although this approach was first proposed as a revisited version of the Space 

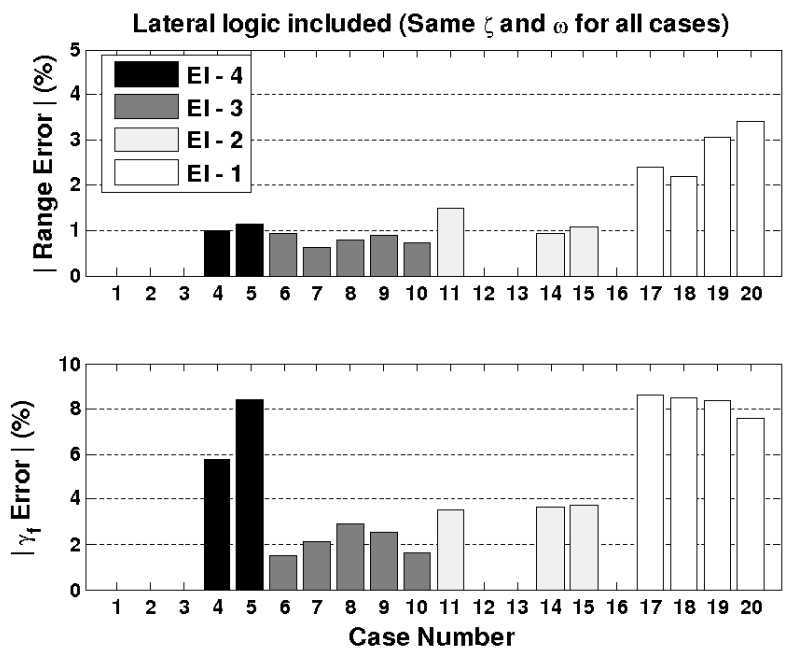

Figure 12. Absolute Range and Final Flight Path Angle Errors of the Controlled Trajectories When the Lateral Logic is Included. The Errors are with Respect to the Values of the 20 Nominal Skip Entry Cases Flown Using NSEG.

Shuttle Orbiter entry guidance to demonstrate the commonality of both guidance laws, it has never been used to fly a skip-like entry trajectory, where, at least for the first entry, the entire drag profile does not fit a quadratic polynomial of the velocity. This approach has been combined with an Apollo-like lateral logic, and satisfactory results were achieved when the gains in the error dynamics were changed at a certain point along the trajectory that is dependent on the velocity and the curvature of the drag as a function of the velocity.

A guidance law that includes an integral feedback term, as is the case in the actual Space Shuttle entry guidance and as is proposed in Ref 6 , could be tried in future studies to assess whether its use results in an improvement of the tracking performance, and to evaluate the design needs when determining the control gains. The introduction of dispersions and the capacity to update the drag profile in flight should be studied as well in future works. Also, future studies should attempt to link the guidance for the first entry, as presented in this paper, with a more standard concept for the second entry, such as the Apollo entry guidance, to try to assess the overall skip entry performance.

\section{Appendix A}

When we deal with the five basic equations required to generate a drag reference profile, the drag polynomial must have degree $4(m=4)$ to result in five unknown coefficients $\left(a_{0}, \ldots, a_{4}\right)$. The equation on range, Eq. (20), implies that numerical methods need to be used to find at least one of the coefficients in the drag polynomial. By using the other four equations, each coefficient can be expressed in terms of one coefficient of our choice. In this way, only that one coefficient shall have to be determined through numerical methods. If, for instance, $a_{3}$ is the coefficient selected, $a_{0}, a_{1}, a_{2}$ and $a_{4}$ can be expressed in terms of $a_{3}$.

To simplify the expressions, the following definition is going to be made

$$
\Delta^{(n)} V \equiv V_{f}^{n}-V_{i}^{n}, n>1
$$

The system of four algebraic linear equations (range equation not included) is given by 


$$
\left(\begin{array}{ccccc}
1 & V_{i} & V_{i}^{2} & V_{i}^{3} & V_{i}^{4} \\
1 & V_{f} & V_{f}^{2} & V_{f}^{3} & V_{f}^{4} \\
0 & 1 & 2 V_{i} & 3 V_{i}^{2} & 4 V_{i}^{3} \\
0 & 1 & 2 V_{f} & 3 V_{f}^{2} & 4 V_{f}^{3}
\end{array}\right)\left(\begin{array}{c}
a_{0} \\
a_{1} \\
a_{2} \\
a_{3} \\
a_{4}
\end{array}\right)=\left(\begin{array}{c}
D_{i} \\
D_{f} \\
D_{i}^{\prime} \\
D_{f}^{\prime}
\end{array}\right)
$$

Doing the necessary operations in Eq. (52) the following equations result for the coefficients $a_{0}, a_{1}, a_{2}$ and $a_{4}$

$$
\begin{aligned}
& a_{4}=a_{4_{1}}+a_{4_{2}} a_{3} \\
& a_{2}=a_{2_{1}}+a_{2_{2}} a_{3} \\
& a_{1}=a_{1_{1}}+a_{1_{2}} a_{3} \\
& a_{0}=a_{0_{1}}+a_{0_{2}} a_{3}
\end{aligned}
$$

where

$$
\begin{aligned}
& a_{4_{1}}=\left(\Delta D^{\prime}\left(2 V_{i}-\Delta^{(2)} V / \Delta V\right)+2 \Delta D-2 D_{i}^{\prime} \Delta V\right) / k \\
& a_{4_{2}}=-\left(3 \Delta^{(2)} V\left(2 V_{i}-\Delta^{(2)} V / \Delta V\right)-2\left(3 V_{i}^{2} \Delta V-\Delta^{(3)} V\right)\right) / k \\
& k=4 \Delta^{(3)} V\left(2 V_{i}-\Delta^{(2)} V / \Delta V\right)-2\left(4 V_{i}^{3} \Delta V-\Delta^{(4)} V\right) \\
& a_{2_{1}}=\left(\Delta D^{\prime}-4 a_{4_{1}} \Delta^{(3)} V\right) /(2 \Delta V) \\
& a_{2_{2}}=-\left(3 \Delta^{(2)} V+4 a_{4_{2}} \Delta^{(3)} V\right) /(2 \Delta V) \\
& a_{1_{1}}=\left(\Delta D-a_{2_{1}} \Delta^{(2)} V-a_{4_{1}} \Delta^{(4)} V\right) / \Delta V \\
& a_{1_{2}}=-\left(a_{2_{2}} \Delta^{(2)} V+\Delta^{(3)} V+a_{4_{2}} \Delta^{(4)} V\right) / \Delta V \\
& a_{0_{1}}=D_{i}-a_{1_{1}} V_{i}-a_{2_{1}} V_{i}^{2}-a_{4_{1}} V_{i}^{4} \\
& a_{0_{2}}=-\left(a_{1_{2}} V_{i}+a_{2_{2}} V_{i}^{2}+V_{i}^{3}+a_{4_{2}} V_{i}^{4}\right)
\end{aligned}
$$

With these results, the problem has been reduced to find numerically the coefficient $a_{3}$ that satisfies the system formed by the range equation in terms of time (Eq. (20)) and the root equation for the drag polynomial. Using this method, the resulting drag reference profile was generated for 4 of the 20 nominal cases considered in this study, one for each entry interface. Those profiles are depicted in figure 13.

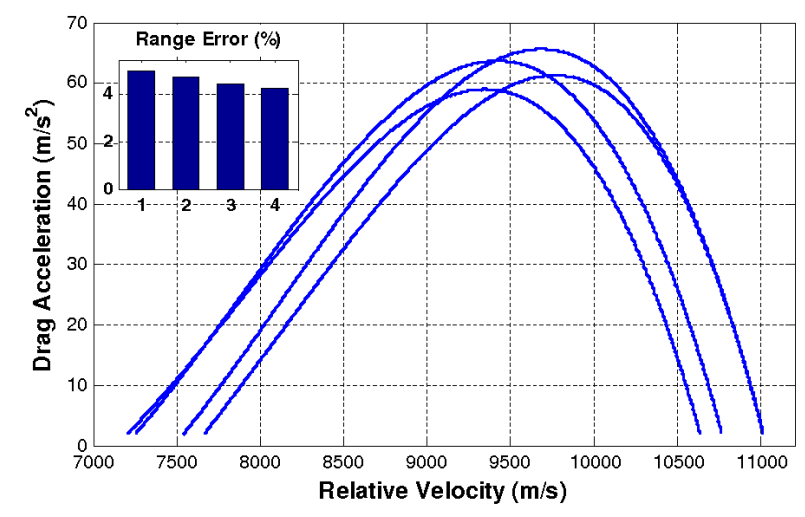

Figure 13. Four Drag Reference Trajectories, One from Each Entry Interface, Generated Using the Range Eq. (20), and Resulting Absolute Range Errors with Respect to the Actual Ranges of the Corresponding Test Nominal Skip Entry Cases Flown Using NSEG. 


\section{Appendix B - Roots of the Drag Polynomial}

This section intends to provide some insight into why the drag polynomial resulting from Eq. (31) happens to be positive in the interval of interest $\left[V_{i}, V_{f}\right]$. Specifically, the following analysis is going to be focused on a degree 5 drag polynomial. The conclusions from this case can be easily applied to the case of a degree 4 polynomial.

All the drag curves found have shown to have three real roots and two imaginary roots. Figure 14 shows the drag curves outside the interval of interest.

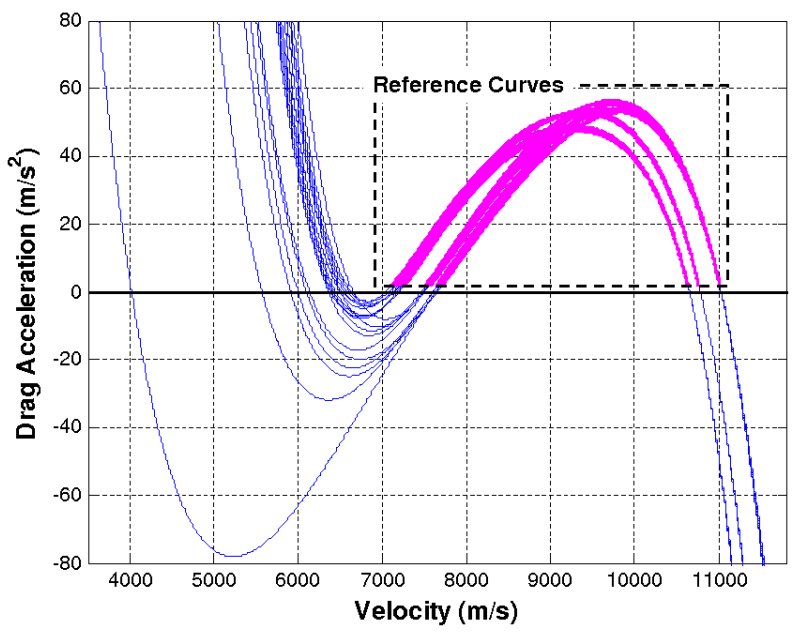

Figure 14. Drag Reference Curves Extended Outside the Interval of Interest.

The drag polynomial of degree 5 can be expressed as

$$
D(V)=\left(V-V_{i}\right)\left(V-V_{f}\right) P_{3}(V)+D_{i}
$$

where two roots of the drag evaluated at the initial and final drag values $\left(D_{f}=D_{i}\right)$ are the initial and final velocities, and where $P_{3}(V)$ stands for a generic polynomial of degree 3. A polynomial of odd degree, such as $P_{3}(V)$, always has at least one real root, which in our case we will call $V_{r}$. Therefore, the drag polynomial can rewritten as

$$
D(V)=\left(V-V_{i}\right)\left(V-V_{f}\right)\left(V-V_{r}\right) x_{2}\left(V^{2}+x_{1} V+x_{0}\right)+D_{i}
$$

If $V_{r}$ is forced to be smaller than $V_{f}$, as was the case with the 20 runs considered, then the signs of all factors, but one, in the interval $\left[V_{i}, V_{f}\right]$ can be determined. Let's say $V_{r}=V_{f}-y^{2}$, then

$$
D(V)-D_{i}=\overbrace{\left(V-V_{i}\right)}^{-} \overbrace{\left(V-V_{f}\right)}^{+} \overbrace{\left(V-V_{f}+y^{2}\right)}^{+} x_{2}\left(V^{2}+x_{1} V+x_{0}\right)
$$

Hence, to have the drag defined as a positive polynomial in the interval of interest the sign of $x_{2}\left(V^{2}+x_{1} V+x_{0}\right)$ must be negative, at least in that interval. The fact that the initial and final flight path angles are negative and positive respectively means that $D_{i}^{\prime}<0$ and $D_{f}^{\prime}>0$ and therefore

$$
\begin{aligned}
& D^{\prime}\left(V_{i}\right)=\overbrace{\left(V_{i}-V_{f}\right)}^{+} \overbrace{\left(V-V_{f}+y^{2}\right)}^{+} x_{2}\left(V^{2}+x_{1} V+x_{0}\right)<0 \\
& D^{\prime}\left(V_{f}\right)=\overbrace{\left(V_{f}-V_{i}\right)}^{-} \overbrace{\left(V-V_{f}+y^{2}\right)}^{+} x_{2}\left(V^{2}+x_{1} V+x_{0}\right)>0
\end{aligned}
$$

which implies that, at least, in the bounds of the interval $\left[V_{i}, V_{f}\right]$ the factor $x_{2}\left(V^{2}+x_{1} V+x_{0}\right)$ is actually forced to be negative to satisfy the imposed conditions on the flight path angle. This conclusion does not demonstrate that the drag is necessarily positive within the interval of interest but, at least, provides some insight into what happens in the cases examined. 
Two conditions that must be met in order to have $x_{2}\left(V^{2}+x_{1} V+x_{0}\right)$ defined negative are: first, $x_{2}<0$ and second, the roots of $V^{2}+x_{1} V+x_{0}$ must be imaginary, which implies that its two roots will be complex conjugates. The second condition implies that $x_{0}^{2}>x_{1}^{2} / 4$ and therefore the drag polynomial with the condition to be positive with no real roots in $\left[V_{i}, V_{f}\right]$ can be rewritten generically as

$$
D(V)=\left(V-V_{i}\right)\left(V-V_{f}\right)\left(V-V_{f}+y^{2}\right)\left(-x_{2}^{2}\right)\left(V^{2}+x_{1} V+x_{1}^{2} / 4+x_{0}^{2}\right)+D_{i}
$$

Equation 59 accounts for the roots at $V_{i}$ and $V_{f}$ at $D=D_{i}=D_{f}$ and hence only four coefficients have to be found, one for each of the remaining equations.

\section{Acknowledgments}

The author wants to thank Christopher J. Cerimele and Lee E. Bryant, respectively Branch Chief and Deputy Chief for the Flight Mechanics and Trajectory Design Branch at the NASA's Johnson Space Center, for their interest in this work. I also want to thank Jeremy R. Rea, of the Orion CEV NSEG team at the Flight Mechanics and Trajectory Design Branch, for providing the data I requested, and to Juan S. Senent, Odyssey Space Research LLC contractor to the Flight Mechanics and Trajectory Design Branch, for his comments.

\section{References}

\footnotetext{
${ }^{1}$ Tigges, M. et. al., "Numerical Skip-Entry Guidance," Guidance and Control Conference, Breckenridge, CO, February 3-7, 2007 , also AAS Paper 07-076.

${ }^{2}$ Harpold, J. C. and Graves, C. A., "Shuttle Entry Guidance," Journal of the Astronautical Sciences, Vol. 27, No. 3, 1979, pp. 239-268.

${ }^{3}$ Mease, K. D. and Kremer, J-P., "Shuttle Entry Guidance Revisited Using Nonlinear Geometric Methods," Journal of Guidance, Control and Dynamics, Vol. 17, No. 6, November-December 1994.

${ }^{4}$ Papoulis A., Probability, Random Variables, and Stochastic Processes, 3rd ed., McGraw-Hill, Inc., 1991.

${ }^{5}$ Moseley, P. E., "The Apollo Entry Guidance: A Review of the Mathematical Development and Its Operational Characteristics", TRW Note No. 69-FMT-791, TRW, December 1, 1969.

${ }^{6}$ Roenneke, A. J. and Well, K. H., "Non-Linear Drag Tracking Control Applied to Optimal Low-Lift Reentry Guidance," Guidance, Navigation and Control Conference, San Diego, CA, July 29-31, 1996 , also AIAA Paper 96-3698.
} 